\title{
The histological significance of atypical glandular cells on cervical cytology: Experience at Groote Schuur Hospital, Cape Town, South Africa
}

\author{
L D Hoffman, ${ }^{1}$ MSc; H-T Wu, ${ }^{2}$ MB BCh, MMed, FCPath, FRCPath \\ ${ }^{1}$ MB ChB III candidate, Faculty of Health Sciences, University of Cape Town, South Africa \\ ${ }^{2}$ Division of Anatomical Pathology, Department of Pathology, Faculty of Health Sciences, University of Cape Town; South African Medical \\ Research Council Gynaecological Cancer Research Centre, Department of Obstetrics and Gynaecology, Faculty of Health Sciences, University of \\ Cape Town; and D7 Anatomical Pathology Laboratory, Groote Schuur Hospital/National Health Laboratory Service, Cape Town, South Africa
}

Corresponding author: L D Hoffman(hoffmanld@yahoo.com)

Background. Atypical glandular cells (AGC) identified on Pap tests may be markers for potentially significant pathology.

Objectives. Primarily, to correlate AGC findings at Groote Schuur Hospital (GSH), Cape Town, South Africa, with subsequent histological investigations and attempt to identify predictors of pathology relevant to the clinical management of women with a cytological diagnosis of AGC. Secondly, to compare the GSH data with data from similar international studies.

Methods. Records of AGC Pap tests were retrieved from the laboratory database in the anatomical pathology laboratory at GSH and clinically relevant information was summarised based on the available information. Standard descriptive statistics were used to summarise the study data, and Fisher's exact test was used to compare categorical outcomes, where possible.

Results. Of the 237 women with a cytological diagnosis of AGC and who had subsequent histological diagnoses, 120 (50.6\%) had significant pathology (cervical intraepithelial neoplasia (CIN) 2 or worse). Significant cervical pathology was most common in women aged $<50$ years, while significant endometrial pathology predominated in women aged $\geq 50$ years. The results of the GSH study were largely consistent with international findings, but the risk of malignancy was six times higher in the GSH population than in a comparable international group. Conclusion. AGC identified on Pap tests may be markers for potentially significant pathology. Human papillomavirus DNA testing is recommended for younger women diagnosed with AGC to reduce invasive investigations and minimise expenses in a resource-poor setting.

S Afr Med J 2016;106(9):907-911. DOI:10.7196/SAMJ.2016.v106i9.10472

The 2001 Bethesda System for Reporting Cervical Cytology ${ }^{[1]}$ represents the latest international consensus for classifying cervical cytology. In terms of this typology, the finding 'atypical glandular cells' (AGC) indicates 'morphological changes that exceed those normally expected but that are not sufficient to suggest a diagnosis of adenocarcinoma in situ' (AIS). ${ }^{[1]}$

AGC is an uncommon cytological interpretation, occurring in $<0.5 \%$ of routine Pap tests. ${ }^{[2-4]}$ Given its rarity and the manifold sources of glandular cells, an AGC diagnosis is associated with a high level of interobserver variability and manifold differential diagnoses ${ }^{[5]}$ However, a cytological diagnosis of AGC is associated with an increased risk of premalignant and invasive lesions. ${ }^{[2,6,7]}$ To our knowledge, there have been no studies published in the English language literature correlating AGC classification with histological diagnosis in South African (SA) patients. Our study may therefore be relevant in the SA context, given that cervical carcinoma remains the most common cancer of women in Africa, with an age-standardised mortality rate of $21.0 \%$ compared with a worldwide mortality rate of $8.9 \%{ }^{[8]}$ SA is characterised by a high prevalence of HIV infection and low cervical screening rates. ${ }^{[8]}$ Current research estimates that the proportion of HIV-positive patients in Cape Town undergoing at least one Pap test is $13.1 \%{ }^{[8]}$ while $<10 \%$ of HIV-negative women utilise the available screening programmes. ${ }^{[9]}$

\section{Objectives}

Primarily, to correlate definitive histological findings following a suspicious Pap test diagnosed as AGC and to summarise these findings, given the available data. Secondly, to compare the data from Groote Schuur Hospital (GSH), Cape Town, with similar international studies ${ }^{[10]}$ with the intention of comparing local findings with other published results. Throughout the study, patterns that may improve management of AGC and/or suggest future research, especially in low-resource settings, were sought.

\section{Methods}

A retrospective audit of all Pap tests diagnosed as AGC on cervical cytology and subsequent histological investigations performed by the National Health Laboratory Service at GSH was undertaken for the period 17 February 2003 - 31 December 2014.

Similar audits as well as studies that explored the clinical significance of AGC were identified using a Medline/PubMed search of the English literature from 1988 to 2015. The search criteria included, but were not limited to, the terms 'atypical glandular cells', 'AGC, 'AGUS, 'atypical glandular cells and HPV' and 'management of atypical glandular cells'.

The total number of patients who received Pap tests over the period 2003 - 2014 was estimated by extracting all Pap test reports from the laboratory information software, Disa ${ }^{\star}$ Lab (Laboratory System Technologies, SA) in the anatomical pathology laboratory at GSH and then merging the records with the same identity numbers. Where identity numbers were unavailable, Pap test results that shared hospital number and date of birth were merged to form a single record. Records with no associated histological investigations (167/424) were removed, as were samples deemed insufficient by the pathologist $(9 / 424)$ and entries with no recorded age (1/424). Recurrent AGC diagnoses over the period of investigation were present (10/424), and 
these records were merged and identified accordingly. Following this procedure, there were 237 patients with AGC cytological diagnoses and corresponding histological diagnoses suitable for analysis. In many cases, information regarding HIV status, menopausal classification, postmenopausal bleeding, contraception method, parity, sample adequacy, reason for Pap test, presence of pathogens and condition of the cervix at time of Pap test were selectively recorded. In cases where menopausal status was not indicated, the age of 50 years was considered to be a proxy for menopause. In addition, if patient records indicated postmenopausal bleeding but no menopausal status was provided, these women were inferred to be postmenopausal.

Where histological results relating to the same cytological finding described different pathology in a uterine or extrauterine site at different points in time, these pathologies were recorded in the order of their diagnosis. However, for the purposes of allocating a distinct diagnostic label to each patient, the most clinically significant diagnosis was used. Lesions classified histologically as normal, benign or inflammatory were grouped together. Discrete diagnostic classifications were created for low-grade squamous intraepithelial lesions (LSIL), highgrade squamous intraepithelial lesions (HSIL), AIS, cervical carcinoma, endometrial hyperplasia $(\mathrm{EH})$, endometrial carcinoma and other (extrauterine) malignancy. Significant pathology was defined to mean a histological diagnosis of CIN2+ (CIN2/3/ AIS), $\mathrm{EH}$ or carcinoma.

The current protocol at GSH does not include routine testing for human papillomavirus (HPV) genotypes. However, the diagnosis of HSIL and any form of cervical carcinoma strongly suggests infection with high-risk HPV, ${ }^{[4]}$ and where these diagnoses

Table 1. Histological diagnoses in patients
with a cytological diagnosis of AGC
\begin{tabular}{ll}
\hline Histological diagnosis & $\boldsymbol{n}(\%)$ \\
\hline Benign & $82(36.4)$ \\
LSIL & $35(14.8)$ \\
HSIL (CIN2+) & $40(16.9)$ \\
Cervical AIS & $2(0.8)$ \\
Cervical Ca. & $28(11.8)$ \\
Endometrial Ca. & $47(19.8)$ \\
EH & $2(0.8)$ \\
Vaginal Ca. & $1(0.4)$ \\
Total & $237(100.0)$ \\
Ca. $=$ carcinoma. &
\end{tabular}

were available they were used as a proxy for infection with high-risk HPV.

The data analysed are included below. Where differences in proportions of categorical variables were computed, Fisher's exact test was used. Comparisons with a $p$-value of $<0.05$ were deemed to be statistically significant. Statistical analysis was performed using R version 2.15.3 (R Foundation for Statistical Computing, Austria). Ethics approval was obtained from the Human Research Ethics Committee, University of Cape Town (HREC Ref: 406/2015)

\section{Results}

An estimated 410867 patients received Pap tests over the study period, of which 424 (0.1\%) were classified as AGC on cervical cytology. Table 1 sets out the corresponding histological diagnoses recorded for the 237 cases that were suitable for analysis.

Of the 237 women diagnosed with AGC on cervical cytology, 120 (50.6\%) were diagnosed with significant clinical disease. Importantly, 76 of the diagnoses in this group (32.1\% of the total) were classified as malignancies.

Of AGC diagnoses at GSH, 86 lesions (36.3\%) were found to be squamous in origin on histological examination, as opposed to 61 glandular lesions (25.7\%).
There was one case of extrauterine pathology (0.4\%), namely vaginal malignancy. Seven of the uterine carcinomas (3.0\% of the total) were identified histologically as Müllerian mixed tumours.

Data from this study were compared with aggregated data from 24 international studies $^{[10]}$ using similar diagnostic criteria. We summarise this comparison in Table 2.

\section{Age}

The mean age of patients in the study was 50.5 years (standard deviation 13.6, median 49.0 , range $25-91)$; 120 women $(50.6 \%)$ were aged $<50$ years and $117(49.4 \%)$ $\geq 50$ years. The age distribution of women with an AGC diagnosis and subsequent histological correlation at GSH is shown in Fig. 1. A linear trend line was superimposed for endometrial carcinoma $\left(R^{2}=0.84\right)$, while polynomial regression was used to fit lines for HSIL $\left(R^{2}=0.72\right)$ and cervical carcinoma $\left(R^{2}=0.93\right)$.

In the GSH group, the incidence of CIN2+ lesions peaked at age 40 years (mean 41.8 ), while the prevalence of cervical carcinoma was highest at 49 years (mean 50.1) and that of endometrial carcinoma at 65 years (mean 65.2). An age of $\geq 50$ years was predictive of significant pathology (odds ratio (OR) 2.39,

Table 2. Comparison of the histological findings of AGC Pap tests referenced in aggregated international data ${ }^{[10]}$ with data collected at GSH from 2003 to 2014

\begin{tabular}{llll}
\hline Outcome & International data, \% (range) $)^{[10]}$ & GSH data, \% & Variance \\
\hline Prevalence of AGC & $0.3(0.1-6.0)$ & 0.1 & 0.2 \\
AGC Pap with histology & $57.0(36.3-100.0)$ & 56.0 & 1.0 \\
AIS & $2.9(0.0-15.7)$ & 0.8 & 2.0 \\
EH & $1.4(0.0-11.8)$ & 0.8 & 0.5 \\
Malignancy & $5.2(0.0-15.5)$ & 32.1 & 26.9 \\
HSIL & $11.1(1.5-46.7)$ & 16.9 & 5.8 \\
LSIL & $8.5(1.2-16.7)$ & 14.8 & 6.3
\end{tabular}

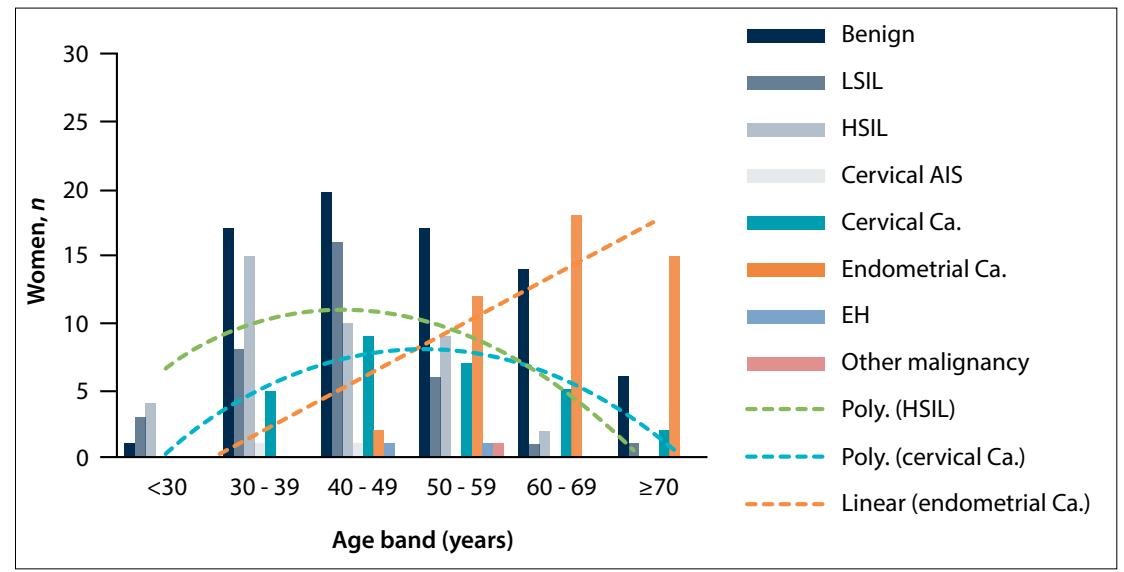

Fig. 1. Histological diagnosis by age band in women with a cytological diagnosis of AGC. (Ca. = carcinoma; Poly. = polynomial regression.) 
$95 \%$ confidence interval 1.38 - 4.12; $p<0.001$ ). Fig. 2 summarises the distribution of histological diagnoses in women aged $<50$ years and those aged $\geq 50$ years.

In addition to the age data, quantitative information regarding menopausal classification, postmenopausal bleeding, recurrent AGC diagnoses, HIV status, condition of the cervix, sample adequacy, presence of the transformation zone (TZ), reason for the Pap test, presence of non-HIV/HPV pathogens, parity and contraception method was collected. Results of these findings are summarised in Tables 3 and 4. In each table only outcomes classified as benign, HSIL, AIS, cervical or endometrial carcinoma are detailed explicitly. The column headed 'Other Dx.' presents the residuals for all diagnoses that did not fall into the groups previously specified. Each risk group was broken down as a percentage of total AGC Pap tests $(N=237)$ and followed by a breakdown of histological diagnoses according to the number of women per risk category.

Menopausal status and postmenopausal bleeding. Histological outcomes according to menopausal status and postmenopausal bleeding are set out in Table 3.

Recurrent AGC diagnoses. Recurrent AGC diagnoses appeared to increase the risk of significant pathology (OR 1.48), but this increase was not statistically significant.

Condition of cervix, classification of sample and absence of the TZ. The clinical condition of the cervix as 'suspected carcinoma' appeared to be a predictor of endometrial carcinoma in the study population. Likewise, the classification of a histological sample as unsatisfactory appeared to be predictive of endometrial carcinoma, as did absence of the TZ.

HPV infection. Seventy women (29.5\%) were diagnosed with HSIL or cervical malignancy and were therefore suspected to be positive for high-risk HPV DNA.
Presence of non-HIV/HPV sexually transmitted pathogens. Bacterial infection or the suggested presence of Candida species, Gardnerella vaginalis, herpes simplex virus, Chlamydia or Trichomonas vaginalis was recorded. The prevalence of HSIL or cervical carcinoma was $31.9 \%$ in women infected with at least one pathogen v. $29.1 \%$ in noninfected women.

Parity. The prevalence of HSIL or cervical carcinoma in multiparous women was $32.3 \%$ v. $16.7 \%$ in women reported to have borne at most one child.

Contraception. The prevalence of HSIL or cervical carcinoma was $45.9 \%$ among women in the GSH group using hormonal contraception v. $38.1 \%$ in women using no contraception.

\section{Discussion}

In the GSH population of women reported to have AGC on cervical cytology, histological findings confirmed significant pathology in the majority of cases.

Women aged $\geq 50$ years were 2.39 times more likely to have significant pathology than women aged $<50$ years. Other predictive factors were postmenopausal bleeding, recurrent AGC diagnoses, HIV infection, the presence of sexually transmitted pathogens, multiparity, hormonal contraception, absence of the TZ and suspicion of carcinoma on macroscopic assessment of the cervix.

Age is recognised as an important risk factor in the development of significant pathology ${ }^{[4]}$ The GSH data reflect the natural histories of both cervical and endometrial carcinomas, with the latter being nearly 23 times more prevalent in the study population after 50 years of age. Furthermore, the GSH data are aligned with reports suggesting that the development of cervical/endometrial carcinoma takes several years to decades to progress from preneoplastic disease to invasive carcinoma. ${ }^{[4,11]}$ The ratio of squamous to glandular lesions appears consistent with that reported in the literature. ${ }^{[2,4,11]}$

Postmenopausal bleeding has been shown to predict invasive pathology in women aged $>50$ years. ${ }^{[2]}$ These findings were confirmed in the GSH population, where women with

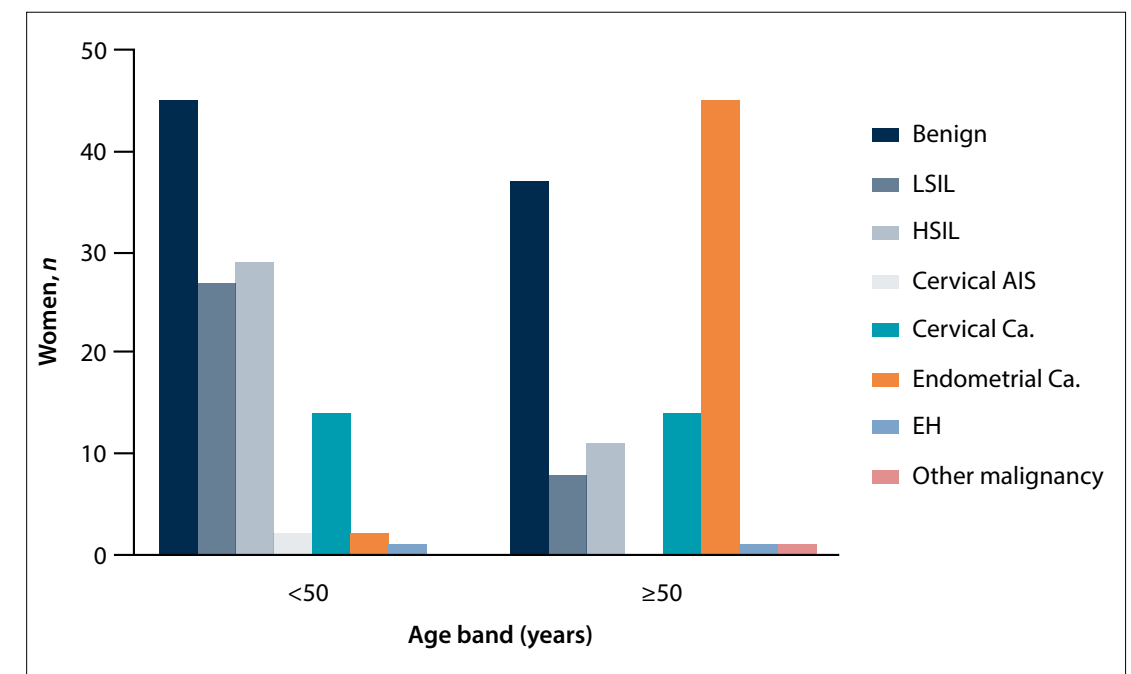

Fig. 2. Histological diagnosis by age band ( $<50$ and $\geq 50$ years) in women with a cytological diagnosis of AGC. (Ca. = carcinoma.)

Table 3. Histological outcomes in women with a cytological diagnosis of AGC, according to menopausal status and postmenopausal bleeding

\begin{tabular}{|c|c|c|c|c|c|c|c|}
\hline Risk factor & $\begin{array}{l}\text { AGC Dx. } \\
(N=237), \%\end{array}$ & Benign, \% & HSIL, \% & AIS, \% & Cerv Ca., \% & Endo Ca., \% & Other Dx., \% \\
\hline \multicolumn{8}{|l|}{ Menopausal status } \\
\hline Premenopausal & 48.5 & 18.1 & 11.8 & 0.8 & 5.9 & 0.0 & 63.4 \\
\hline Postmenopausal & 51.5 & 32.0 & 9.8 & 0.0 & 11.5 & 38.5 & 8.2 \\
\hline \multicolumn{8}{|c|}{ Postmenopausal bleeding } \\
\hline Present & 11.0 & 46.2 & 0.0 & 0.0 & 3.8 & 42.3 & 7.7 \\
\hline Absent & 3.8 & 11.1 & 66.7 & 0.0 & 11.1 & 11.1 & 0.0 \\
\hline NA & 85.2 & 34.2 & 16.8 & 1.0 & 12.9 & 17.3 & 17.8 \\
\hline
\end{tabular}


Table 4. Histological outcomes in women with a cytological diagnosis of AGC, according to Pap test, HIV status, presence of nonHIV/HPV pathogens, parity and method of contraception

\begin{tabular}{|c|c|c|c|c|c|c|c|}
\hline Risk factor & $\begin{array}{l}\text { AGC Dx. } \\
(N=237), \%\end{array}$ & Benign, \% & HSIL, \% & AIS, \% & Cerv Ca., \% & Endo Ca., \% & Other Dx., \% \\
\hline \multicolumn{8}{|c|}{ Recurrent AGC diagnosis } \\
\hline Yes & 4.2 & 30.0 & 20.0 & 0.0 & 20.0 & 20.0 & 10.0 \\
\hline No & 95.8 & 34.8 & 16.7 & 0.9 & 11.5 & 19.8 & 16.3 \\
\hline \multicolumn{8}{|l|}{ Cervical condition } \\
\hline Healthy & 43.0 & 43.1 & 15.7 & 2.0 & 7.8 & 17.6 & 13.8 \\
\hline Inflammatory & 9.3 & 36.4 & 27.3 & 0.0 & 18.2 & 9.1 & 9.0 \\
\hline Suspect Ca. & 7.6 & 22.2 & 0.0 & 0.0 & 22.2 & 50.0 & 5.6 \\
\hline NA & 40.1 & 27.4 & 18.9 & 0.0 & 12.6 & 18.9 & 22.2 \\
\hline \multicolumn{8}{|l|}{ Adequacy } \\
\hline Satisfactory & 85.7 & 36.5 & 17.2 & 1.0 & 11.8 & 16.7 & 16.8 \\
\hline Unsatisfactory & 10.1 & 25.0 & 16.7 & 0.0 & 16.7 & 37.5 & 4.1 \\
\hline NA & 4.2 & 20.0 & 10.0 & 0.0 & 0.0 & 40.0 & 30.0 \\
\hline \multicolumn{8}{|l|}{$\mathrm{TZ}$} \\
\hline Present & 92.8 & 35.9 & 17.3 & 0.9 & 11.8 & 17.3 & 16.8 \\
\hline Absent & 5.5 & 7.7 & 15.4 & 0.0 & 15.4 & 61.5 & 0.0 \\
\hline NA & 1.7 & 50.0 & 0.0 & 0.0 & 0.0 & 25.0 & 25.0 \\
\hline \multicolumn{8}{|l|}{ Reason for Pap test } \\
\hline Screening & 31.6 & 32.0 & 26.7 & 2.7 & 10.7 & 10.7 & 17.2 \\
\hline Diagnostic & 26.2 & 43.5 & 6.5 & 0.0 & 11.3 & 25.8 & 12.9 \\
\hline NA & 42.2 & 31.0 & 16.0 & 0.0 & 13.0 & 23.0 & 17.0 \\
\hline \multicolumn{8}{|l|}{ HIV } \\
\hline Positive & 6.8 & 31.2 & 43.8 & 6.2 & 18.8 & 0.0 & 0.0 \\
\hline Negative & 22.4 & 45.3 & 13.2 & 0.0 & 5.7 & 24.5 & 11.3 \\
\hline NA & 70.9 & 31.5 & 15.5 & 0.6 & 13.1 & 20.2 & 19.1 \\
\hline \multicolumn{8}{|c|}{ Non-HIV/HPV pathogens } \\
\hline Present & 29.1 & 33.3 & 29.0 & 0.0 & 2.9 & 10.1 & 24.7 \\
\hline Absent & 67.9 & 35.4 & 12.4 & 1.2 & 15.5 & 23.6 & 11.9 \\
\hline NA & 3.0 & 28.6 & 0.0 & 0.0 & 14.3 & 28.6 & 28.5 \\
\hline \multicolumn{8}{|l|}{ Multiparity } \\
\hline Yes & 14.3 & 38.2 & 17.6 & 0.0 & 14.7 & 20.6 & 8.9 \\
\hline No & 2.5 & 33.3 & 0.0 & 0.0 & 16.7 & 0.0 & 50.0 \\
\hline NA & 83.1 & 34.0 & 17.3 & 1.0 & 11.2 & 20.3 & 16.2 \\
\hline \multicolumn{8}{|l|}{ Contraception } \\
\hline Condom & 0.4 & 100.0 & 0.0 & 0.0 & 0.0 & 0.0 & 0.0 \\
\hline None & 26.6 & 28.6 & 17.5 & 0.0 & 20.6 & 23.8 & 9.5 \\
\hline Hormonal & 10.1 & 37.5 & 37.5 & 4.2 & 4.2 & 0.0 & 16.6 \\
\hline Tubal ligation & 2.5 & 83.3 & 16.7 & 0.0 & 0.0 & 0.0 & 0 \\
\hline NA & 60.3 & 34.3 & 13.3 & 0.7 & 9.8 & 22.4 & 19.5 \\
\hline
\end{tabular}

postmenopausal bleeding were three times more likely to have significant pathology than postmenopausal women who were not experiencing such bleeding.

The prevalence of HIV in SA is estimated to be $12.2 \%$ (5.0\% in the Western Cape) ${ }^{[12]}$ so the incomplete data in respect of HIV status are likely to include several HIV-positive women. Patients with HIV have an increased absolute risk of an AGC diagnosis. ${ }^{[3]}$ Furthermore, HIV-positive women are five times more likely to be infected with HPV than
HIV-negative women, ${ }^{[3,9]}$ which provides a plausible explanation for the higher incidence of squamous cervical lesions in the GSH group.

It is generally agreed that HPV is the agent that causes cervical neoplasia. ${ }^{[4,9,13]}$ There are 
over 100 recognised genotypes of HPV, but types 16 and 18 account for approximately $70 \%$ of cases of CIN. ${ }^{[13]}$ Research also suggests that HPV DNA testing is useful to differentiate between cervical and endometrial lesions ${ }^{[4]}$ and that infection with HPV increases a woman's risk of cervical pathology significantly. ${ }^{[9]}$ Local studies in women with atypical cervical cytology have estimated the prevalence of high-risk HPV at $54.3 \% \cdot{ }^{[14]}$ The estimated prevalence of high-risk HPV in the GSH data would therefore be expected to be closer to the reported local prevalence, and this represents a serious limitation in the analysis of the GSH data.

The GSH data point to an increased risk of HPV-associated cervical lesions in women infected with sexually transmitted pathogens. Research suggests that co-infection with HPV and other sexually transmitted diseases increases the risk of cervical carcinoma. ${ }^{[9]}$ In the GSH study, the risk of HSIL or cervical carcinoma in women with an AGC diagnosis was higher among those who tested positive for sexually transmitted pathogens.

There was an increased prevalence of HSIL and cervical carcinoma among multiparous women in the GSH study, which is consistent with published research. ${ }^{[9]}$

Continuous use of oral contraceptives for $>5$ years has been shown to increase the risk of significant cervical pathology. ${ }^{[9]}$ Women in the GSH study using hormonal contraception did have an increased prevalence of such pathology, but any further inference was limited given that the duration of use of hormonal contraceptives was not recorded on the database. The explanation for the increase in endometrial carcinoma in women not using contraception is probably age-related, given that postmenopausal women do not require contraception.

The study findings suggest that the prevalence of AGC in the GSH population is within the range described in the international literature. ${ }^{[10]}$ The notably higher proportion of malignancies in the GSH data is probably a result of current protocol regarding Pap testing in the public health system, coupled with a high burden of HIV/HPV infection in SA. Currently the National Department of Health offers asymptomatic women three free Pap tests at 10-year intervals from 30 years of age. ${ }^{[9,15]}$ It is clear, however, that most SA women have never had a Pap test, ${ }^{[9]}$ and this lack of population-based screening and treatment facilitates long-term HPV infection, which poses the single largest risk for cervical carcinoma ${ }^{[15]}$ and probably explains the elevated comparative risk of malignancy encountered in poor countries, ${ }^{[9]}$ as observed in the GSH data. In low-resource settings such as SA, where utilisation of Pap tests is low, HPV genotyping should be considered as a screening method, especially in younger women, given that absence of high-risk HPV DNA renders the risk of significant disease negligible for some time. ${ }^{[15,16]}$ This would facilitate longer screening intervals, with an associated reduction in screening costs and benefits to patients.

Research has shown that the sensitivity of HPV DNA testing is equivalent to that of cytological screening and that it may be easier to implement. ${ }^{[17]}$ In this regard, the introduction of point-of-care HPV DNA testing using the GeneXpert (Cepheid, USA) cartridge-based system holds some promise. This qualitative real-time polymerase chain reaction assay tests for 14 high-risk HPV genotypes in about 1 hour and exhibits clinical performance comparable to current batch HPV DNA testing, which takes several hours to complete. ${ }^{[18]}$ As a consequence of SA's high prevalence of HIV and a burdened public health system, the incidence of tuberculosis and HPV is high. ${ }^{[18]}$
The National Department of Health has embarked on a national programme for detecting tuberculosis using the GeneXpert MTB/RIF assay. ${ }^{[18]}$ The infrastructure already in place could be simultaneously deployed to run GeneXpert HPV assays as a primary screening tool or an adjunct to cervical cytology. ${ }^{[18]}$ While this proposal merits further investigation with regard to cost and feasibility, point-of-care testing would be beneficial in terms of reducing losses to follow-up in the SA public health sector, where the turnaround time for a Pap test is currently around 6 weeks.

\section{Conclusion}

In the GSH setting, AGC is a rare but important finding, indicating significant pathology in a majority of cases, with rates of malignancy higher than those found in comparable international investigations. ${ }^{[10]}$ The findings of the GSH study suggest that when AGC is diagnosed in women aged $>50$ years, colposcopy and endometrial sampling are indicated. However, in women diagnosed with AGC aged $<50$ years and who are HIV- and HPV-negative, more conservative management could be considered in the form of more frequent Pap tests and/or HPV genotyping rather than immediate colposcopy.

Acknowledgement. The authors are grateful for valuable input from Prof. L Denny, head of the Gynaecological Cancer Research Centre (funded by the South African Medical Research Council).

1. Solomon D, Davey D, Kurman R, et al. The 2001 Bethesda System: Terminology for reporting results of Solomon D, Davey D, Kurman R, et al. The 2001 Bethesda System: Terminology for
cervical cytology. JAMA 2002;287(16):2114-2119. DOI:10.1001/jama.287.16.2114

cervical cytology. JAMA 2002;287(16):2114-2119. DOI:10.1001/jama.287.16.2114
Schorge JO, Rauh-Hain JA. Atypical glandular cells. Clin Obstet Gynecol 2013;56(1):35-43. Schorge JO, Rauh-Hain JA. Atypic
DOI:10.1097/GRF.0b013e3182823849

3. Massad LS, Xie X, Darragh TM, et al. Histologic correlates of glandular abnormalities in cervical cytology among women with human immunodeficiency virus. Obstet Gynecol 2009;114(5):10631068. DOI:10.1097/AOG.0b013e3181bc6ce0

4. Castle PE, Fetterman B, Poitras N, Lorey T, Shaber R, Kinney W. Relationship of atypical glandular cell cytology, age, and human papillomavirus detection to cervical and endometrial cancer risks. Obste Gynecol 2010;115(2):243-248. DOI:10.1097/AOG.0b013e3181c799a3

5. Confortini M, di Bonito L, Carozzi F, et al. Interlaboratory reproducibility of atypical glandular cells of undetermined significance: A national survey. Cytopathology 2006;17(6):353-360. DOI:10.1111/ j.1365-2303.2006.00372.x

6. Duska LR. Can we improve the detection of glandular cervical lesions: The role and limitations of the Pap smear diagnosis atypical glandular cells (AGC). Gynecol Oncol 2009;114(3):381-382. DOI:10.1016/j.ygyno.2009.07.008

7. Zhao C, Florea A, Onisko A, Austin RM. Histologic follow-up results in 662 patients with Pap Zhao C, Florea A, Onisko A, Austin RM. Histologic follow-up results in 662 patients with Pap
test findings of atypical glandular cells: Results from a large academic women's hospital laboratory test findings of atypical glandular cells: Results from a large academic womens hospital laboratory
employing sensitive screening methods. Gynecol Oncol 2009;114(3):383-389. DOI:10.1043/2008-

0755-OAR1.

8. Batra P, Kuhn L, Denny L. Utilisation and outcomes of cervical cancer prevention services among HIVinfected women in Cape Town. S Afr Med J 2010;100(1):39-44

9. Denny L. Prevention of cervical cancer. Reprod Health Matters 2008;16(32):18-31. DOI:10.1016 S0968-8080(08)32397-0

0. Schnatz PF, Guile M, O'Sullivan DM, Sorosky JI. Clinical significance of atypical glandular cells on cervical cytology. Obstet Gynecol 2006;117(3):701-708. DOI:10.1097/01.AOG.0000202401.29145.68

1. Wang SS, Sherman ME, Hildesheim A, et al. Cervical adenocarcinoma and squamous cell carcinom incidence trends among white women and black women in the United States for 1976-2000. Cancer 2004;100(5):1035-1044. DOI:10.1002/cncr.20064

12. Shisana O, Rehle T, Simbayi LC, et al. South African National HIV Prevalence, Incidence and Behaviour Survey, 2012. Cape Town: HSRC Press, 2014:xxiii-xxiv.

13. Kumar V, Abbas AK, Aster J, eds. Robbins Basic Pathology. 9th ed. Philadelphia: Elsevier, 2013:685689.

14. Richter K, Becker P, Horton A, Dreyer G. Age-specific prevalence of cervical human papillomavirus infection and cytological abnormalities in women in Gauteng Province, South Africa. S Afr Med 2013;103(5):313-317. DOI:10.7196/SAMJ.6514

15. South African HPV Advisory Board. Cervical cancer and human papillomavirus: South African guidelines for screening and testing. S Afr J Gynaecol Oncol 2010;2(1):23-26.

16. Crothers JW, Mount SL, Harmon M, Wegner E. The utility of human papillomavirus testing in young women with atypical glandular cells on Pap test. J Low Genit Tract Dis 2015;19(1):22-26. DOI:10.1097/ LGT.0000000000000044

17. Kuhn L, Denny L, Pollack A, Lorincz A, Richart RM, Wright TC. Human papillomavirus DNA testing for cervical cancer screening in low-resource settings. J Natl Cancer Inst 2000; 92(10):818-825. DOI: $10.1093 /$ inci/92.10.818

18. Einstein MH, Smith KM, Davis TE, et al. Clinical evaluation of the cartridge-based GeneXpert human papillomavirus assay in women referred for colposcopy. J Clin Microbiol 2014;52(6):2089-2095. DOI:10.1128/JCM.00176-14

Accepted 21 June 2016 